\title{
Avaliação de Custo-efetividade nas terapias oncológicas, emprego de modelos de Markov: Uma revisão integrativa da literatura
}

\author{
Cost-effectiveness evaluation in cancer therapies, use of Markov models: An integrative \\ literature review
}
Evaluación de Costo-efectividad en las terapias oncológicas, empleo de modelos de Markov: Una revisión literaria integradora

José Afonso Corrêa da Silva ${ }^{1 *}$.

\begin{abstract}
RESUMO
Objetivo: Revisar a literatura científica que aborda a relação custo-efetividade das terapias oncológicas, correlacionado com o limitar de disposição de pagar e qualidade de vida (QALYs). Métodos: Foi realizada uma revisão da literatura através da busca de publicações nos bancos de dados PUBMED, LILACS e EMBASE, em outubro de 2018. A estratégia de pesquisa consistiu em: termos para "cost effectiveness"; para "chemotherapy"; para "cancer"; e, associados ao termo "markov model". Resultados: A pesquisa resultou em 209 publicações, dos quais ao final, 18 artigos compuseram essa revisão. Na maioria dos trabalhos, o modelo de Markov foi focado no estadiamento da doença, dividindo em três ciclos, Estado livre de progressão (PFS), Doença progressiva (DP) e Morte. Nas avaliações de custo-efetividade poucas terapias apresentaram melhorias no QALYS e um custo, sendo que as comparações que obtiveram, forma de terapias em que as drogas padrão já apresentavam um custo elevado, diminuindo o ICER da terapia inovadora. Conclusão: Os resultados da revisão apontaram uma notável dificuldade de justificar a inserção de novas terapias através dos estudos de custo-efetividade, assim como uma grande diferença entre o limite de disposição a pagar dos diferentes países, o que influencia diretamente na possibilidade de aquisição de novos tratamentos.
\end{abstract}

Palavras-chave: Custo-efetividade, Antineoplásicos, Modelos de Markov.

\begin{abstract}
Objective: Review the scientific literature that addresses the cost-effectiveness of oncologic therapies, correlated with the limit of willingness to pay and quality of life (QALYs). Methods: A review was carried out through the search of publications in PUBMED, LILACS and EMBASE databases, in October 2018. The research strategy consisted of: terms for "cost effectiveness"; for "chemotherapy"; for "cancer"; and, associated with the term "markov model". Results: The research resulted in 209 publications, of which at the end, 18 articles composed this review. In most studies, the Markov model was focused on staging the disease, dividing it into three cycles, Progression Free State (PFS), Progressive Disease (PD) and Death. In the costeffectiveness evaluations, few therapies presented improvements in the QALYS and a cost, and the comparisons that they obtained, form of therapies in which the standard drugs already presented a high cost, reducing the ICER of the innovative therapy. Conclusion: The results of the review showed a notable difficulty in justifying the insertion of new therapies through cost-effectiveness studies, as well as a great difference between the willingness to pay of the different countries, which directly influences the possibility of acquiring new treatments.
\end{abstract}

Key words: Cost-effectiveness, Antineoplastics, Markov Models.

${ }^{1}$ Universidade Federal do Rio Grande do Sul, Porto Alegre - RS. *E-mail: joseafonso.hsvp@gmail.com 


\section{RESUMEN}

Objetivo: Repasar la literatura científica que aborda la relación costo-efectividad de las terapias oncológicas, correlacionada con el limitar de disposición de pagar y calidad de vida (QALYs). Métodos: Se realizó una revisión a través de la búsqueda de publicaciones en los bancos de datos MEDLINE, LILACS y EMBASE, en octubre de 2018. La estrategia de investigación consistió en: términos para "rentabilidad"; para la quimioterapia; para el cáncer; y, asociados al término "markov model". Resultados: La encuesta resultó en 209 publicaciones, de las cuales al final, 18 artículos compusieron esa revisión. En la mayoría de los trabajos, el modelo de Markov se centró en la estadificación de la enfermedad, dividiendo en tres ciclos, estado libre de progresión (PFS), enfermedad progresiva (DP) y muerte. En las evaluaciones de costo-efectividad pocas terapias presentaron mejoras en el QALYS y un costo, siendo que las comparaciones que obtuvieron, forma de terapias en que las drogas estándar ya presentaban un costo elevado, disminuyendo el ICER de la terapia innovadora. Conclusión: Los resultados de la revisión apuntaron una notable dificultad de justificar la inserción de nuevas terapias a través de los estudios de costo-efectividad, así como una gran diferencia entre el límite de disposición a pagar de los diferentes países, lo que influye directamente en la posibilidad de adquisición de nuevas terapias nuevos tratamientos.

Palabras clave: Costo-efectividad, Antineoplásicos, Modelos de Markov.

\section{INTRODUÇÃO}

O câncer é de fato uma grande ameaça à saúde pública, tanto no âmbito social quando no econômico, contabilizando aproximadamente 596 mil novos casos no ano de 2016 (INCA, 2016), classificando-o como sendo a segunda maior causa de morte no Brasil e no mundo (WHO, 2015). A alta incidência das doenças é impulsionada principalmente pelo aumento do envelhecimento da população.

O Fórum Econômico Mundial estimou custos diretos de tratamento de câncer e compareceu a valores de perdas de renda em US\$ 290 bilhões em 2010 (BLOOM DE, et al., 2011), resultando na União Europeia, por exemplo, em custos para o tratamento do câncer maiores que 126 milhões de euros (LUENGO-FERNANDEZ $\mathrm{R}$, et al., 2013). Dentro os motivos para o crescimento dos gastos na área, certamente encontramos o investimento em pesquisa de rastreio avançado, diagnóstico precoce e nas modalidades de tratamento, este fato, não deixam dúvida de que os custos do câncer só se expandirão (BLOOM DE, et al., 2011). No Brasil, as despesas com tratamentos de câncer (cirurgia oncológica, radioterapia, quimioterapia e iodoterapia) cresceu 357\% entre os anos de 2000 e 2014 (BRASIL, 2015).

Além disso, a consciência do impacto psicológico para os pacientes e a importância de manter a saúde relacionada qualidade de vida (QVRS) durante e após a terapia antineoplásica, têm aumentado nas últimas décadas (BOTTOMLEY A, 2002). Nos campos da oncologia cirúrgica e radiológica, grandes progressos já foram feitos, resultando em tratamentos com menos efeitos colaterais e sobrecarga do paciente.

As modalidades de tratamento sistêmico estão de certa forma atrasadas neste aspecto do tratamento. Apesar das evoluções no sentido de quimioterapias com doses mais baixas e menos tóxicas, imunoterapias direcionadas e a alta frequência desses tratamentos, são emocionalmente estressantes para os pacientes e interferem com o seu funcionamento social (HALL M E LLOYD H, 2008).

Neste contexto, é que está inserido a necessidade de desenvolver estudos de custo-efetividade (CE), buscando justificativas para aprovação ou revogação de novas terapias, custosas para os órgãos pagadores e danosas para os pacientes em uso. A avaliação de CE geralmente ocorre por meio de estudos de modelagem comparando duas ou mais coortes com intervenções diferentes, que depois de montar ou utilizar dados de ensaios clínicos já finalizados, é caracterizada e aplicada em uma população hipotética (SECOLI SR, et al., 2010).

Numerosos parâmetros são inseridos no modelos, não se limitando aos custos de terapia, mas sim, probabilidades de doença remanescente/livre de progressão (usualmente baseado em dados), probabilidades de toxicidade (com os custos de gestão de acordo), e os resultados clínicos esperados até a morte (também baseado em dados clínicos e / ou extrapolação dos mesmos) (SECOLI SR, et al., 2010). 
Nesse contexto, os modelos mais empregados, que avaliam a probabilidade de passagem de um estado de saúde a outro são os modelos de Markov, onde o padrão das transições de estado permanecem constantes ao longo do tempo (SATO RC E ZOUAIN DM, 2010) e semi-Markov, onde é possível considerar interferentes no tempo de passagem de um estado a outro (HETTLE R, et al., 2015).

Neste modelo, os custos e resultados são compilados para todos os grupos na duração dentro de cada fase modelada, finalizando no tempo total de avaliação (referido como o horizonte de tempo); os braços são comparados para os custos totais, anos de vida ajustados pela qualidade (QALYs), e os custos associados que cada um ano ganhou de QALY (denominado razão de custo-efetividade incremental "incremental costeffectiveness ratio" - ICER) (SOAREZ PC e NOVAES HMD, 2017).

A ICER indica quanto o pagamento é necessário para um ano adicional de vida (com qualidade) e é comparado com um período de tempo pré-determinado. Esse valor é correlacionado com o limiar de "disposição a pagar" (willingness-to-pay-WTP) que difere por publicação, sociedade, sistema econômico, período de tempo e outros fatores (os limites mais comuns variam de US $\$ 50.000$ e US\$150.000/QALY). A comparação do ICER com o limiar WTP, conclui se a intervenção é ou não considerado "custo-efetivo" (SOAREZ PC E NOVAES HMD, 2017).

Sendo assim, essa revisão tem como objetivo, revisar a literatura cientifica, que aborda a relação custo efetividade das terapias oncológicas, e analisar os valores referentes da mesma, correlacionado com o limitar de disposição de pagar e qualidade de vida (QALYs).

\section{MÉTODOS}

Foi realizada uma revisão integrativa da literatura, baseada nos itens exigidos para revisões e metaanálises PRISMA (PRISMA, Reporting Items for Systematic Reviews and Meta-Analyses) (MOHER D, et al., 2010).

\section{Critérios de elegibilidade}

A elegibilidade da primeira fase de pesquisa foi baseada em todos os artigos que descreveram o emprego de modelos de Markov para a estimativa de custo-efetividade na comparação de protocolos antineoplásicos, estes foram os critérios de inclusão para a presente revisão.

Foram excluídos os artigos, que após selecionados na pesquisa inicial, apresentaram na leitura geral do texto, que apenas citavam o modelo de Markov, ainda, foram excluídos os artigos que traçavam uma comparação entre métodos não farmacológicos, procedimentos, metodologias preventivas, e que faziam avaliações de custo efetividade pela comparação de doses diferentes, fugindo do escopo principal da revisão. Estudos que analisaram a relação entre pacientes de países diferentes, ou até de dois ensaios clínicos diferentes, também foram excluídos.

Visando padronizar os dados para a avaliação de custo-efetividade, foram elegidos apenas artigos que contemplavam todas as informações quanto ao QALYS, ICER e WTP. O mesmo se aplica para editoriais, artigos de opinião, discussões e revisões referentes a iniciativas existentes.

Todos artigos prospectivos randomizados e não randomizados, bem como retrospectivos que analisaram as diferenças no âmbito do custo-efetividade entre terapias farmacológicas no câncer, foram incluídos na revisão da literatura. Não houve nenhuma restrição de idioma. Apenas as publicações dos últimos cinco anos foram consideradas para a revisão.

\section{Estratégia de busca}

Os dados foram sistematicamente identificados através da revisão eletrônica dos bancos de dados MEDLINE, através do acesso pelo PubMed (https://www.ncbi.nlm.nih.gov/pubmed/), LILACS (http://lilacs.bvsalud.org/en/) e EMBASE, através do site do Science Direct (https://www.sciencedirect.com) em outubro de 2018. 
A estratégia de pesquisa consistiu em: (1) termos para "cost effectiveness"; (2) termos para "chemotherapy" (3) termos para "cancer"; (4) associados ao termo "markov model". Os termos correspondentes no MeSH (https://www.ncbi.nlm.nih.gov/mesh), bem como as consultas que foram feitas, estão descritas no Quadro 1.

Quadro 1 - Estratégia de pesquisa.

\begin{tabular}{|c|c|c|c|}
\hline & Cost effectiveness & Chemotherapy & Cancer \\
\hline \multirow[t]{10}{*}{ Mesh } & $\begin{array}{l}\text { a. Cost-Benefit Analyses } \\
\text { [Subheading] }\end{array}$ & a. Drug Therapy [Mesh] & $\begin{array}{l}\text { a. Neoplasms } \\
\text { [Mesh] }\end{array}$ \\
\hline & $\begin{array}{l}\text { b. Cost-Effectiveness } \\
\text { Analysis [Subheading] }\end{array}$ & $\begin{array}{l}\text { b. Consolidation Chemotherapy } \\
\text { [Mesh] }\end{array}$ & b. Cancer \\
\hline & c. Cost effectiveness & c. Induction Chemotherapy [Mesh] & \\
\hline & & d. Chemotherapy, Adjuvant [Mesh] & \\
\hline & & e. Maintenance Chemotherapy [Mesh] & \\
\hline & & f. Drug Therapy, Combination [Mesh] & \\
\hline & & g. Antineoplastic Agents [Mesh] & \\
\hline & & h. Drug Therapy, Combination [Mesh] & \\
\hline & & i. Drug therapy [Subheading] & \\
\hline & & j. Chemotherapy & \\
\hline MEDLINE & (1) $A-C / O R$ & (2) A-J/OR & (3) $A-B / O R$ \\
\hline LILACS & (1) $\mathrm{C}$ & (2) $\mathrm{J}$ & (3) $\mathrm{B}$ \\
\hline EMBASE & (1) $\mathrm{A}-\mathrm{C} / \mathrm{OR}$ & (2) A-J/OR & (3) $A-B / O R$ \\
\hline
\end{tabular}

Fonte: Silva JAC, 2018.

A estratégia de busca foi inicialmente criada para o MEDLINE e posteriormente adaptado para os outros bancos de dados. Os termos com escopo mais amplo, que não se enquadravam no objeto da pesquisa foram descartados, por fugirem em demasia do assunto abordado. A estratégia de busca foi focada apenas em estudos humanos e repetida semanalmente durante a preparação do manuscrito, a fim de identificar e incluir publicações relevantes e potencialmente novas. Uma base de dados bibliográfica foi criada manualmente para armazenar e gerenciar as referências recuperadas através do programa Microsoft Excel 2016.

\section{Avaliação do risco de viés}

A avaliação da qualidade dos estudos selecionados foi realizada juntamente com a extração de dados. A impossibilidade de cegar o pesquisador resultou em um risco de viés de desempenho, assim como, devido a limitações metodológicas, todos os artigos eram propensos ao viés de detecção sendo que a análise não passou por revisão de pesquisadores externos. Para alguns artigos, era impossível julgar todos os domínios da avaliação de risco, pois haviam dados insuficientes disponíveis para permitir julgamento. Ao final, 5 (cinco) artigos foram excluídos, pois o risco de viés foi considerado como sendo significativo.

A qualidade metodológica dos estudos selecionados foi avaliada por um pesquisador, em caso de dúvida, foi solicitado conselho a um segundo pesquisador. Os estudos selecionados eram heterogêneos em termos de desenho de estudo. Portanto, todos os estudos incluídos foram avaliados de acordo com o manual de avaliação Cochrane de ECRs (5.1.0) (HIGGINS JP, et al., 2011).

Os parâmetros incluem: geração de sequência aleatória, alocação e ocultação, cegueira do paciente, pessoal e avaliadores de resultados, resultados incompletos, relatórios e outros vieses. Foi julgado cada item em três níveis ("1" para um viés baixo, "2" para um alto risco de vieses e "3" para não claro). Em seguida, foi organizado os artigos pelos níveis de vieses encontrados, como: baixo risco de vieses (todos os itens foram categorizados em "Sim"), risco de viés (pelo menos um item foi confirmado como "Não") e risco de viés pouco claro (pelo menos um item foi classificado como "Pouco claro"). Para aqueles artigos que avaliaram os parâmetros de custo-efetividade baseando-se em dados de ensaios clínicos randomizados de fase II e III, foram considerados como de baixo risco de vieses. 


\section{Análise de dados}

Após a remoção das duplicatas, foram selecionados artigos relevantes com base no título e resumo. Os artigos selecionados foram selecionados de forma independente da relevância, pelo autor principal (J.A.C), de acordo com os critérios de elegibilidade propostos.

Todos os dados relevantes foram tabulados para criar uma síntese descritiva das informações existentes em cada artigo. Durante a fase de elegibilidade, os estudos tabulados no programa Microsoft Excel 2016, seguiram os seguintes critérios: autor/ano, país, neoplasia, terapias comparadas e etapas do moledo de Markov utilizadas, despesas totais por doente (média), valor o QALYs, valor do ICER e valor de "disposição a pagar".

Ressalta-se que a qualidade de vida requer dados fechados sobre o sofrimento; ansiedade ou depressão; bem-estar, atividades diárias e reações adversas da cada terapia, sendo assim, foram utilizados os valores descritos de cada estudo. Da mesma forma, a comparação do valor "disposto a pagar" com o ICER de cada estudo, foi relacionada de acordo com a nacionalidade pesquisa, mantendo a moeda escolhida para a descrição dos valores monetários.

\section{RESULTADOS}

\section{Resultado da pesquisa}

A fase de identificação resultou em 209 publicações. Após a retirada das duplicatas 196 artigos foram selecionados para a fase de triagem. Duas publicações foram incluídas na revisão nesta fase. Sendo assim, 198 artigos passaram pela triagem de título e resumo, dos quais 50 foram retidos para a revisão do texto completo, na fase de elegibilidade. As principais razões da exclusão dos textos completos estão listadas no diagrama PRISMA (Figura 1).

Figura 1 - Diagrama PRISMA para critérios de exclusão e composição da amostra.

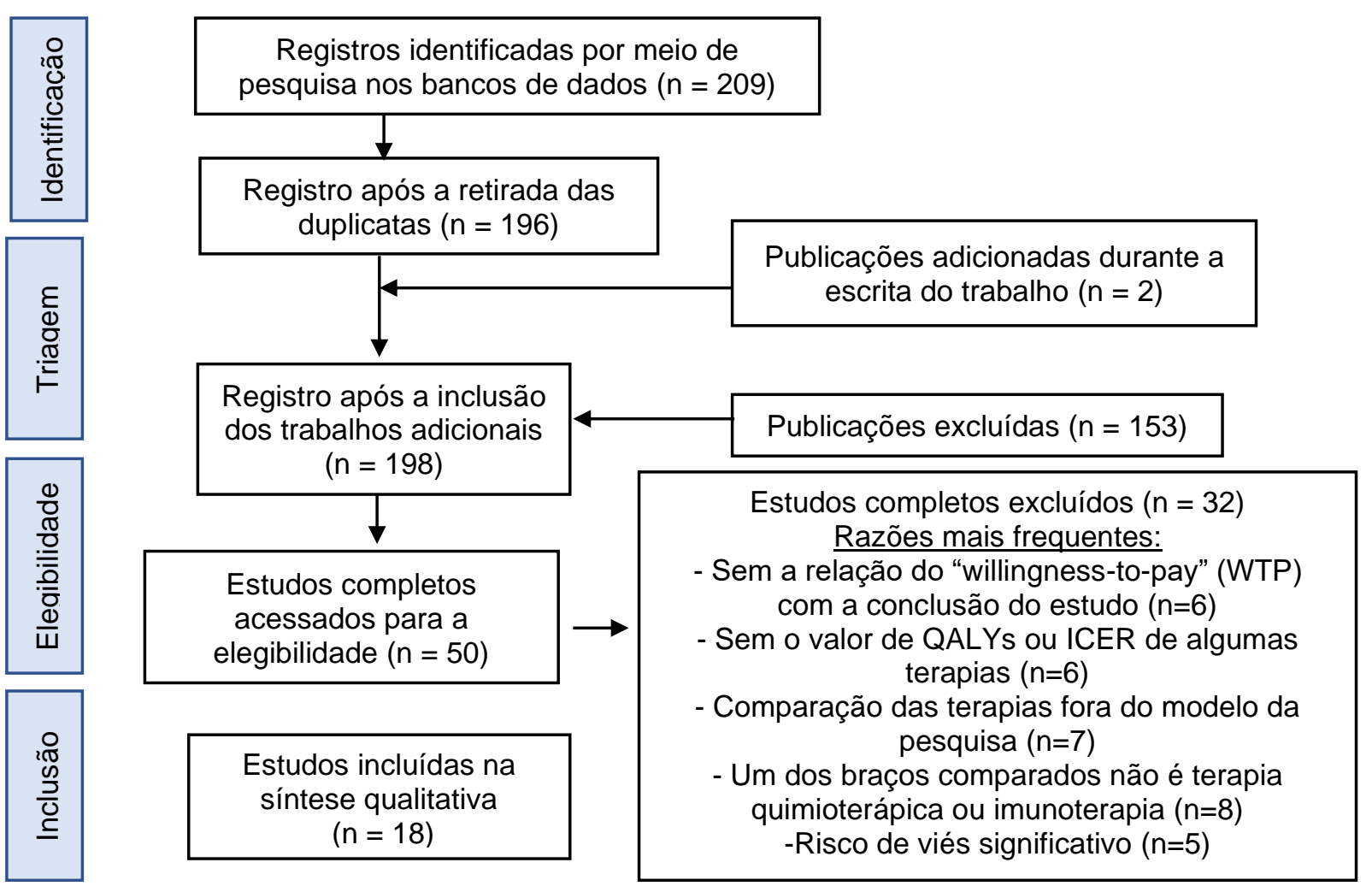

Fonte: Silva JAC, 2018. 
Listam-se abaixo: Artigos que obtiverem seus dados de ensaios clínico de fase II/III: [Zhou K, et al., 2018 - (JCOG0605); Zhen H, et al., 2018 - (BEYOND); Zhang P, et al., 2016 - (ORIENTAL, EACH); Zhan M, et al., 2017 - (MAPS); Xie J, et al., 2015 - (BOLERO-2); Sarfaty M, et al., 2018 - (CheckMate 025 e RECORD-1); Saito S, et al., 2017 - (RAINBOW); Rivera F, et al., 2017 - (PEAK); Miguel LS, et al., 2017 - (KN-006); MatterWalstra K, et al., 2015 - (ClinicalTrials.gov numbers, NCT01584648 e NCT01597908); Leal L, et al., 2018 (COU-AA-301); Hu X e Hay JW, 2018 - (KEYNOTE-024); Goldstein DA, et al., 2015 -(SQUIRE); Cohn DE, et al., 2015 - (GOG 218); Carlson JJ, et al., 2017 - (ASCEND I e II); Bohensky MA, et al., 2016- (CA209066)]; Artigo que extraiu os dados para um dos braços do estudo, de um ensaio clínico de fase III: [Van Kampen RJW, et al., 2017 - (E2100)].

\section{Revisão literária dos resultados de interesse}

Ao ler esta revisão, deve-se ter em mente que os modelos e métodos dos estudos abordados em sua grande maioria, baseiam-se em ensaios clínicos randomizados, porém com avaliações custo efetivas heterogêneos, utilizadas para explorar os desfechos de interesse; especialmente, em relação as diferentes nacionalidades que cada estudo apresenta, o que gera mudanças importantes nos critérios econômicos e comparativos. O tamanho amostral que compõe as avaliações e o horizonte temporal de cada avaliação econômica, não foram incluídos nesta revisão, devido à grande diferença nos mesmos, sendo que cada estudo utilizado e neoplasia avaliada carece, nesses dois pontos, de critérios individualizados. Uma visão geral das conclusões feitas em cada documento, referente relação custo-efetividade das terapias comparas, é dada nos Quadros 2 e 3.

\section{Modelo de Markov}

Na maioria dos trabalhos o modelo de Markov foi focado no estadiamento da doença, dividindo em três ciclos, Estado livre de progressão (PFS), Doença progressiva (DP) e Morte, utilizando uma semana Miguel LS, et al. (2017) ou um mês para o tempo de passagem entre cada estado, prazos condizentes a evolução da doença e/ou resposta ao tratamento (SARFATY M, et al., 2018; ZHEN H, et al., 2018; ZHOU K, et al., 2018).

Em alguns trabalhos o modelo de Markov, foi construído com base em um número maior de etapas e com tempo de passagem diferente, como de duas semanas Rivera F, et al. (2017), além disso, alguns autores optaram por configurar o modelo de acordo com a terapia utilizada, ampliando assim o número de fases (GOLDSTEIN DA, et al., 2015).

Em alguns casos, os autores descreveram que utilizaram softwares para a elaboração do modelo como TreeAge Saito S, et al. (2017); Zhan M, et al. (2017); Zhou K, et al. (2018) ou Microsoft Excel (CARLSON JJ, et al., 2017).

\section{Mensuração dos custos}

Os estudos pesquisados avaliaram os custos totais na perspectiva do pagador de saúde do país de origem, no mesmo foi incluído duas partes: custos médicos diretos e custos sociais, ou indiretos. O primeiro cobre 0 custo de medicamentos, da administração dos mesmos, exames, tratamentos para Eventos adversos (EAs) e hospitalização Casado LF, et al. (2018); Sarfaty M, et al. (2018); Zhen H, et al. (2018) e custos terminais Xie $\mathrm{J}$, et al. (2015), enquanto o segundo se referia a taxas de viagem (fixados por viagem ao hospital) e custos de tempo Zhou K, et al. (2018), assim como os dias em que os pacientes ficaram sem poder trabalhar (ZHANG $P$, et al., 2016).

Em algumas situações, foram avaliados apenas os custos médicos diretos Miguel LS, et al. (2017); Rivera F, et al. (2017); Hu X e Hay JW, (2018), pelo fato de os autores discutirem a perspectiva de pagamento em saúde por terceiros. 


\begin{tabular}{|c|c|c|c|c|c|}
\hline Código & Autor Ano & País & Neoplasia & Terapias comparadas & Etapas do Modelo de Markov \\
\hline A1 & Casado LF, et al., 2018 & Espanha & $\begin{array}{l}\text { Leucemia Linfocítica Crônica } \\
\text { redicivante ou refratária }\end{array}$ & $\begin{array}{l}\text { Idelalisib + Rituximabe Vs. } \\
\text { Rituximabe }\end{array}$ & $\begin{array}{l}\text { 1-Pacientes livres de progressão da doença; } \\
\text { 2- Pacientes com progressão da doença. } \\
\text { 3-Morte. }\end{array}$ \\
\hline A2 & $\begin{array}{l}\text { Matter-Walstra K, et al., } \\
2015\end{array}$ & Suíça & $\begin{array}{l}\text { Melanoma metastático com } \\
\text { mutação no BRAF V600 }\end{array}$ & $\begin{array}{l}\text { Dabrafenibe + Trametinibe } \\
\text { Vs. Vemurafenibe }\end{array}$ & $\begin{array}{l}\text { 1-Pacientes livres de progressão da doença; } \\
\text { 2- Pacientes com progressão da doença. } \\
\text { 3-Morte. }\end{array}$ \\
\hline A3 & Zhen H, et al., 2018 & China & $\begin{array}{l}\text { Câncer de pulmão de células } \\
\text { não-pequenas metastático } \\
\text { não-escamosas cancro. }\end{array}$ & $\begin{array}{l}\text { Bevacizumabe }+ \\
\text { Paclitaxel-carboplatina } \\
\text { Vs. Paclitaxel-Carboplatina }\end{array}$ & $\begin{array}{l}\text { 1-Pacientes livres de progressão da doença; } \\
\text { 2- Pacientes com progressão da doença. } \\
\text { 3-Morte. }\end{array}$ \\
\hline A4 & $\begin{array}{l}\text { Bohensky MA, et al., } \\
2016\end{array}$ & Austrália & 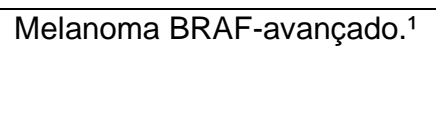 & Nivolumab Vs. Ipilimumab & $\begin{array}{l}\text { 1-Pacientes livres de progressão da doença; } \\
\text { 2- Pacientes com progressão da doença. } \\
\text { 3- Morte. }\end{array}$ \\
\hline A5 & Cohn DE, et al., 2015 & EUA & Câncer de ovário & $\begin{array}{l}\text { Paclitaxel-carboplatina } \\
\text { Vs. Paclitaxel-carboplatina } \\
\text { + Bevacizumab Vs. } \\
\text { Manutenção: Paclitaxel- } \\
\text { carboplatina+Bevacizumab } \\
\text { + Bevacizumab. }\end{array}$ & $\begin{array}{l}\text { 1-Pacientes livres de progressão da doença; } \\
\text { 2- Pacientes com progressão da doença. } \\
\text { 3- Morte. }\end{array}$ \\
\hline A6 & Leal L, et al., 2018 & Brasil & $\begin{array}{l}\text { Câncer de próstata } \\
\text { metastático }\end{array}$ & $\begin{array}{l}\text { Docetaxel Vs. Docetaxel + } \\
\text { Abiraterona }\end{array}$ & $\begin{array}{l}\text { 1-Pacientes livres de progressão da doença; } \\
\text { 2- Pacientes com progressão da doença. } \\
\text { 3- Morte. }\end{array}$ \\
\hline A7 & Sarfaty M, et al., 2018 & Israel & Carcinoma de células renais & $\begin{array}{l}\text { Everolimus Vs. } \\
\text { Nivolumabe }\end{array}$ & $\begin{array}{l}\text { 1-Pacientes livres de progressão da doença; } \\
\text { 2- Pacientes com progressão da doença. } \\
\text { 3- Morte. }\end{array}$ \\
\hline A8 & Miguel LS, et al., 2017 & Portugal & Melanoma avançado & $\begin{array}{l}\text { Pembrolizumabe } \\
\text { Vs. Ipilimumab }\end{array}$ & $\begin{array}{l}\text { 1-Pacientes livres de progressão da doença; } \\
\text { 2- Pacientes com progressão da doença. } \\
\text { 3- Morte. }\end{array}$ \\
\hline A9 & Rivera F, et al., 2017 & Espanha & Câncer colorretal metastático & $\begin{array}{l}\text { Panitumumabe + } \\
\text { mFOLFOX6 }{ }^{2} \\
\text { Vs. Bevacizumab + } \\
\text { mFOLFOX6 } 6^{2}\end{array}$ & $\begin{array}{l}\text { 1- Pacientes livres de progressão da doença; } \\
\text { 2- Doença progressiva: Tratada com o melhor cuidado de suporte; } \\
\text { 3- Doença progressiva: Tratada com terapia ativa subsequente; } \\
\text { 4- Tentativa de ressecção de metástases; } \\
\text { 5- Livre de doença após ressecção de metástases; } \\
\text { 6-Doença progressiva: após ressecção e recidiva; } \\
\text { 7- Morte. }\end{array}$ \\
\hline
\end{tabular}




\section{Revista Eletrônica Acervo Saúde / Electronic Journal Collection Health ISSN 2178-2091}

\begin{tabular}{|c|c|c|c|c|c|}
\hline A10 & Zhan M, et al., 2017 & China & Mesotelioma pleural maligno & $\begin{array}{l}\text { Premetexede/ cisplatina } \\
\text { Vs. Premetexede/ } \\
\text { cisplatina + Bevacizumabe }\end{array}$ & $\begin{array}{l}\text { 1-Pacientes livres de progressão da doença; } \\
\text { 2- Pacientes com progressão da doença. } \\
\text { 3- Morte. }\end{array}$ \\
\hline A11 & Zhou K, et al., 2018 & China & $\begin{array}{l}\text { Câncer de pulmão de células } \\
\text { não pequenas }\end{array}$ & $\begin{array}{l}\text { Cisplatina }+ \text { Etoposide }+ \\
\text { Irinotecano } \\
\text { Vs. Topotecano }\end{array}$ & $\begin{array}{l}\text { 1-Pacientes livres de progressão da doença; } \\
\text { 2- Pacientes com progressão da doença. } \\
\text { 3- Morte. }\end{array}$ \\
\hline A12 & Saito S, et al., 2017. & Japão & Câncer gástrico avançado & $\begin{array}{l}\text { Paclitaxel + Ramucirumab } \\
\text { Vs. Paclitaxel }\end{array}$ & $\begin{array}{l}\text { 1-Pacientes livres de progressão da doença; } \\
\text { 2- Pacientes com progressão da doença. } \\
\text { 3- Morte. }\end{array}$ \\
\hline A13 & Xie J, et al., 2015 & EUA & Câncer de mana & $\begin{array}{l}\text { Exemestano + Everolimus } \\
\text { Vs. Exemestano ou } \\
\text { Fulvestrant ou } \\
\text { Tamoxifeno }\end{array}$ & $\begin{array}{l}\text { 1-Pacientes livres de progressão da doença; } \\
\text { 2- Pacientes com progressão da doença. } \\
\text { 3- Morte. }\end{array}$ \\
\hline A14 & $\begin{array}{l}\text { Hu X and Hay JW, } \\
2018\end{array}$ & $\begin{array}{l}\text { Reino } \\
\text { Unido }\end{array}$ & $\begin{array}{l}\text { Câncer de pulmão avançado } \\
\text { de células não pequenas }\end{array}$ & $\begin{array}{l}\text { Pembrolizumab } \\
\text { Vs. Quimioterapia padrão. }\end{array}$ & $\begin{array}{l}\text { 1-Pacientes livres de progressão da doença; } \\
\text { 2- Pacientes com progressão da doença. } \\
\text { 3- Morte. }\end{array}$ \\
\hline A15 & Zhang P, et al., 2016 & China & Carcinoma hepatocelular & FOLFOX4² Vs. Sorafenib & $\begin{array}{l}\text { 1-Pacientes livres de progressão da doença; } \\
\text { 2- Pacientes com progressão da doença. } \\
\text { 3- Morte. }\end{array}$ \\
\hline A16 & $\begin{array}{l}\text { Goldstein DA, et al., } \\
2015\end{array}$ & EUA & $\begin{array}{l}\text { Câncer de pulmão } \\
\text { metastático }\end{array}$ & $\begin{array}{l}\text { Gemcitabina + Cisplatina. } \\
\text { Vs. Gemcitabina + } \\
\text { Cisplatina + Necitumumab } \\
\text { e manutenção com } \\
\text { Necitumumab. }\end{array}$ & $\begin{array}{l}\text { 1-Tratamento de primeira linha (terapias comparadas) antes da } \\
\text { progressão; } \\
\text { 2- Tratamento de segunda linha após a progressão; } \\
\text { 3- Morte. }\end{array}$ \\
\hline A17 & $\begin{array}{l}\text { Van Kampen RJW, et } \\
\text { al., } 2017\end{array}$ & Holanda & Câncer de Mama & $\begin{array}{l}\text { Bevacizumabe + Taxanos } \\
\text { Vs. Taxanos }\end{array}$ & $\begin{array}{l}\text { 1-Pacientes livres de progressão da doença; } \\
\text { 2- Pacientes com progressão da doença. } \\
\text { 3- Morte. }\end{array}$ \\
\hline A18 & Carlson J, et al., 2017 & EUA & $\begin{array}{l}\text { Câncer avançado de pulmão } \\
\text { de não pequenas células }\end{array}$ & $\begin{array}{l}\text { Alectinibe } \\
\text { Vs. Ceritinibe }\end{array}$ & $\begin{array}{l}\text { 1-Pacientes livres de progressão da doença; } \\
\text { 2- Pacientes com progressão da doença. } \\
\text { 3- Morte. }\end{array}$ \\
\hline
\end{tabular}

Fonte: Silva JAC, 2018.

Legenda: ${ }^{1}$ GRAF: gene B-Raf; mFOLFOX6, ${ }^{2}$ FOLFOX4: regime quimioterápico de 5-Fluorouracil + leucovorina + oxaliplatina. 


\begin{tabular}{|c|c|c|c|c|c|}
\hline Código & Autor Ano & $\begin{array}{l}\text { Despesas totais por } \\
\text { doente (Média) }\end{array}$ & QALYs $^{1}$ & ICER $^{2}$ & $\begin{array}{l}\text { Conclusão por "disposição a pagar" (willingness-to-pay- } \\
\text { WTP) }\end{array}$ \\
\hline A1 & $\begin{array}{l}\text { Casado LF, et } \\
\text { al., } 2018\end{array}$ & $\begin{array}{l}\text { Idelalisib+ Rituximabe } \\
\text { ( } 118.254 \text { euros); } \\
\text { Rituximabe ( } 23.874 \text { euros) }\end{array}$ & $\begin{array}{l}\text { Idelalisib+ } \\
\text { Rituximabe (4.97); } \\
\text { Rituximabe (1.82). }\end{array}$ & $\begin{array}{l}\text { Redução de 15.257euros } \\
\text { por QALY para } \\
\text { Idelalisib+ } \\
\text { Rituximabe }\end{array}$ & $\begin{array}{l}\text { Disposição a pagar }=<50.000 / \text { QALY euros. } \\
\text { Considerando o limite de disposição para pagar, a combinação } \\
\text { de idelalisib+rituximabe é custo-efetivo em } 78 \% \text { das } \\
\text { simulações. }\end{array}$ \\
\hline A2 & $\begin{array}{l}\text { Matter-Walstra } \\
\text { K, et al., } 2015\end{array}$ & $\begin{array}{l}\text { Dabrafenibe }+ \\
\text { Trametinibe } \\
\text { (311.421 francos suíços); } \\
\text { Vemurafenibe } \\
\text { (111773 francos suíços) }\end{array}$ & $\begin{array}{l}\text { Dabrafenibe + } \\
\text { Trametinibe } \\
(1.54) ; \\
\text { Vemurafenibe } \\
\text { (1.02). }\end{array}$ & $\begin{array}{l}\text { Incremento de } 385603 \\
\text { francos suíços por QALY } \\
\text { para Dabrafenibe + } \\
\text { Trametinibe. }\end{array}$ & $\begin{array}{l}\text { Disposição a pagar }=<100.000 / \text { QALY francos suíços. } \\
\text { É necessária uma redução no preço total da terapia combinada } \\
\text { para alcançar uma relação de custo-efetividade aceitável. } \\
\text { Nenhuma das variações testadas nos principais parâmetros, } \\
\text { resultou numa ICER abaixo de um limiar de WTP. }\end{array}$ \\
\hline A3 & $\begin{array}{l}\text { Zhen H, et al., } \\
2018\end{array}$ & $\begin{array}{l}\text { Bevacizumabe }+ \\
\text { Paclitaxel-carboplatina } \\
\text { (112.943 dólares); } \\
\text { Paclitaxel-Carboplatina } \\
\text { (32.171 dólares) }\end{array}$ & $\begin{array}{l}\text { Bevacizumabe }+ \\
\text { Paclitaxel-carboplatina } \\
(1,07) ; \\
\text { Paclitaxel-Carboplatina } \\
(0,80)\end{array}$ & $\begin{array}{l}\text { Incremento de } 299.155 \\
\text { dólares por QALY para } \\
\text { Bevacizumabe + } \\
\text { Paclitaxel-carboplatina. }\end{array}$ & $\begin{array}{l}\text { Disposição a pagar }=<23.970 / \text { QALY dólares. } \\
\text { Apesar de apresentar um aumento no QALY a associação de } \\
\text { Bevacizumabe + } \\
\text { Paclitaxel-carboplatina, excedeu o limite de disposição para } \\
\text { pagar social aceito, não alcançando uma relação de custo- } \\
\text { efetividade aceitável. }\end{array}$ \\
\hline A4 & $\begin{array}{l}\text { Bohensky MA, } \\
\text { et al., } 2016\end{array}$ & $\begin{array}{l}\text { Nivolumab } \\
\text { (178.612 dólares); } \\
\text { Ipilimumab } \\
\text { (138,987 dólares). }\end{array}$ & $\begin{array}{l}\text { Nivolumab }(2.5) \\
\text { Ipilimumab }(1,2)\end{array}$ & $\begin{array}{l}\text { Redução de } 30.475 \\
\text { dólares por QALY para } \\
\text { Nivolumabe. }\end{array}$ & $\begin{array}{l}\text { A disposição em pagar = <35.000/QALY dólares. } \\
\text { O estudo mostrou que a utilização de Nivolumabe é compatíve } \\
\text { com a realidade Australiana, alcançando uma relação de custo- } \\
\text { efetividade aceitável. }\end{array}$ \\
\hline A5 & $\begin{array}{l}\text { Cohn DE, et } \\
\text { al., } 2015\end{array}$ & $\begin{array}{l}\text { Paclitaxel-carboplatina } \\
\text { (4.044 dólares); } \\
\text { Paclitaxel-carboplatina + } \\
\text { Bevacizumab } \\
\text { (43.703 dólares); } \\
\text { Manutenção com Paclitaxel- } \\
\text { carboplatina + Bevacizumab } \\
\text { + Bevacizumab } \\
\text { (122.700 dólares). }\end{array}$ & $\begin{array}{l}\text { Paclitaxel-carboplatina } \\
\text { (1.1); Paclitaxel- } \\
\text { carboplatina }+ \\
\text { Bevacizumab (1.13); } \\
\text { Manutenção com } \\
\text { Paclitaxel-carboplatina + } \\
\text { Bevacizumab } \\
\text { + Bevacizumab (1.25). }\end{array}$ & $\begin{array}{l}\text { Incremento de } 792.380 \\
\text { dólares por QALY para } \\
\text { Manutenção com } \\
\text { Paclitaxel-carboplatina + } \\
\text { Bevacizumab } \\
\text { + Bevacizumab. }\end{array}$ & $\begin{array}{l}\text { A disposição em pagar }=<100.000 / Q A L Y \text { dólares. } \\
\text { O estudo demonstrou que em nenhuma das terapias, o } \\
\text { Bevacizumab é um tratamento custo efetivo, mesmo quando } \\
\text { desconsiderado o impacto na qualidade de vida (QV). Dado } \\
\text { que a inclusão dos escores de QV aumentou o ICER (por mais } \\
\text { de } 150.000 \text { dólares). }\end{array}$ \\
\hline A6 & $\begin{array}{l}\text { Leal L, et al., } \\
2018\end{array}$ & $\begin{array}{l}\text { Docetaxel } \\
(2.895,32 \text { reais); } \\
\text { Docetaxel }+ \text { Abiraterona } \\
\text { (83.295,02 reais). }\end{array}$ & $\begin{array}{l}\text { Docetaxel } \\
(0.763) ; \\
\text { Docetaxel + Abiraterona } \\
(1.045)\end{array}$ & $\begin{array}{l}\text { Incremento de } 284.416 \\
\text { reais por QALY para } \\
\text { Docetaxel + Abiraterona. }\end{array}$ & $\begin{array}{l}\text { A disposição em pagar }=<100.000 / \text { QALY reais. } \\
\text { Em nenhuma das análises de sensibilidade os valores ficaram } \\
\text { abaixo do limite de disposição para pagar. Mesmo quando } \\
\text { variados simultaneamente seis parâmetros, os resultados } \\
\text { seguiram elevados não alcançando uma relação de custo- } \\
\text { efetividade aceitável com a inclusão da Abiraterona ao } \\
\text { tratamento. }\end{array}$ \\
\hline
\end{tabular}




\begin{tabular}{|c|c|c|c|c|c|}
\hline A7 & $\begin{array}{l}\text { Sarfaty M, et } \\
\text { al., } 2018\end{array}$ & $\begin{array}{l}\text { Everolimus } \\
\text { (50.935 dólares); } \\
\text { Nivolumabe } \\
\text { (101.070 dólares). }\end{array}$ & $\begin{array}{l}\text { Incremento de } 0.34 \\
\text { QALY para o } \\
\text { Nivolumabe. }\end{array}$ & $\begin{array}{l}\text { Incremento de } 146.532 \\
\text { dólares por QALY para } \\
\text { Nivolumabe em } \\
\text { comparação ao } \\
\text { Everolimus e } 226197 \\
\text { dólares por QALY para } \\
\text { Nivolumabe em } \\
\text { comparação ao placebo. }\end{array}$ & $\begin{array}{l}\text { A disposição em pagar }=<150.000 / Q A L Y \text { dólares. } \\
\text { Ao limitar a duração máxima do tratamento com nivolumab a } 2 \\
\text { anos reduziu-se o ICER para } 121.788 / Q A L Y \text { dólares. Sendo } \\
\text { assim, apesar da análise ser limitada pela baixa disponibilidade } \\
\text { de dados, revela-se que a inclusão do nivolumab é custo- } \\
\text { efetivo quando comparado ao Everolimus, porém não quando } \\
\text { comparado com o placebo. }\end{array}$ \\
\hline A8 & $\begin{array}{l}\text { Miguel LS, et } \\
\text { al., } 2017\end{array}$ & $\begin{array}{l}\text { Pembrolizumabe } \\
\text { (156,268 euros); } \\
\text { Ipilimumab } \\
\text { ( } 110,034 \text { euros). }\end{array}$ & $\begin{array}{l}\text { Pembrolizumabe } \\
\text { (3.31); } \\
\text { Ipilimumab } \\
\text { (2.33). }\end{array}$ & $\begin{array}{l}\text { Incremento de } 47.221 \\
\text { euros por QALY para } \\
\text { Pembrolizumabe. }\end{array}$ & $\begin{array}{l}\text { A disposição em pagar }=<50.000 / \text { QALY euros. } \\
\text { De acordo com a análise de sensibilidade probabilística } \\
\text { realizada, o Pembrolizumab está associado em } 75 \% \text { dos casos } \\
\text { a um custo por QALY inferior ao disposto a pagar. } \\
\text { Considerando assim que o mesmo é uma alternativa custo- } \\
\text { efetiva para o tratamento de pacientes com melanoma } \\
\text { avançado em Portugal. }\end{array}$ \\
\hline A9 & $\begin{array}{l}\text { Rivera F, et } \\
\text { al., } 2017\end{array}$ & $\begin{array}{l}\text { Panitumumabe }+ \\
\text { mFOLFOX6 } \\
\text { ( } 57.485 \text { euros); } \\
\text { Bevacizumab }+ \text { mFOLFOX6 } \\
\text { (72.203 euros). }\end{array}$ & $\begin{array}{l}\text { Panitumumabe + } \\
\text { mFOLFOX6 }(2.7) \text {; } \\
\text { Bevacizumab }+ \\
\text { mFOLFOX6 }(2.1)\end{array}$ & $\begin{array}{l}\text { Incremento de } 16.567 \\
\text { euros por QALY para } \\
\text { Panitumumabe }\end{array}$ & $\begin{array}{l}\text { A disposição em pagar }=<30.000 / \text { QALY euros. } \\
\text { Os resultados da análise mostraram que o tratamento de } \\
\text { primeira linha com Panitumumabe }+ \text { mFOLFOX } 6 \text { poderia ser } \\
\text { considerado uma opção econômica em comparação ao } \\
\text { Bevacizumabe + mFOLFOX6. }\end{array}$ \\
\hline $\mathbf{A 1 0}$ & $\begin{array}{l}\text { Zhan M, et al., } \\
2017\end{array}$ & $\begin{array}{l}\text { Premetexede/ cisplatina } \\
\text { (20.348 dólares); } \\
\text { Premetexede/ cisplatina + } \\
\text { Bevacizumabe } \\
\text { (101.795 dólares). }\end{array}$ & $\begin{array}{l}\text { Premetexede/ cisplatina } \\
\text { (0.733); Premetexede/ } \\
\text { cisplatina }+ \\
\text { Bevacizumabe }(0.845) .\end{array}$ & $\begin{array}{l}\text { Incremento de } \\
727.202 \text { euros por QALY } \\
\text { para Premetexede/ } \\
\text { cisplatina }+ \\
\text { Bevacizumabe }\end{array}$ & $\begin{array}{l}\text { Disposição a pagar }=<23.970 / \text { QLY dólares. } \\
\text { Em ambas as análises de sensibilidade unidirecional e } \\
\text { sensibilidade probabilística, a ICER excedeu o limite de } \\
\text { disposição para pagamento normalmente aceito. Não } \\
\text { apresentando custo-efetividade para a inclusão do } \\
\text { Bevacizumabe a terapia para Mesotelioma pleural maligno. }\end{array}$ \\
\hline$\overline{A 11}$ & $\begin{array}{l}\text { Zhou K, et al., } \\
2018\end{array}$ & $\begin{array}{l}\text { Cisplatina + Etoposide + } \\
\text { Irinotecano,(18.995 dólares); } \\
\text { Vs. Topotecano } \\
\text { (12.008 dólares). }\end{array}$ & $\begin{array}{l}\text { Cisplatina + Etoposide + } \\
\text { Irinotecano } \\
(0.85) ; \text { Vs. Topotecano } \\
(0.59) \text {. }\end{array}$ & $\begin{array}{l}\text { Incremento de } 26.720 \\
\text { dólares por QALY para a } \\
\text { Cisplatina + Etoposide + } \\
\text { Irinotecano. }\end{array}$ & $\begin{array}{l}\text { Disposição a pagar = <23.970/QALY dólares. } \\
\text { A combinação de quimioterapia com cisplatina, etoposide e } \\
\text { irinotecano não foi uma opção de custo-efetividade para os } \\
\text { pacientes na China. }\end{array}$ \\
\hline A12 & $\begin{array}{l}\text { Saito S, et al., } \\
2017 .\end{array}$ & $\begin{array}{l}\text { Paclitaxel + Ramucirumab } \\
\text { (4.491.989 yen japonês); } \\
\text { Paclitaxel } \\
\text { (621.913 yen japonês). }\end{array}$ & $\begin{array}{l}\text { Paclitaxel + } \\
\text { Ramucirumab,(0.69); } \\
\text { Paclitaxel }(0.60)\end{array}$ & $\begin{array}{l}\text { Incremento de } \\
\text { 43.010.248 yen japonês } \\
\text { por QALY para a } \\
\text { Paclitaxel + } \\
\text { Ramucirumab }\end{array}$ & $\begin{array}{l}\text { Disposição a pagar }=<12.000 .000 / \text { Q ALY yen japoneses. } \\
\text { A adição de ramucirumab a um regime de paclitaxel no } \\
\text { tratamento de segunda linha do câncer gástrico não } \\
\text { apresentou relação de custo efetividade favorável. }\end{array}$ \\
\hline
\end{tabular}




\begin{tabular}{|c|c|c|c|c|c|}
\hline A13 & $\begin{array}{l}\text { Xie J, et al., } \\
2015\end{array}$ & $\begin{array}{l}\text { Exemestano + Everolimus } \\
\text { (258.648 dólares); } \\
\text { Exemestano } \\
\text { (139.740 dólares); } \\
\text { Fulvestrant } \\
\text { (157.749 dólares); } \\
\text { Tamoxifeno } \\
\text { (115.624 dólares). }\end{array}$ & $\begin{array}{l}\text { Exemestano }+ \\
\text { Everolimus }(1.99) ; \\
\text { Exemestano }(1.60) ; \\
\text { Fulvestrant (1.82); } \\
\text { Tamoxifeno (1.69). }\end{array}$ & $\begin{array}{l}\text { Exemestano + } \\
\text { Everolimus tiveram um } \\
\text { incremento de } 139.740 \\
\text { dólares em comparação } \\
\text { com Exemestano; } \\
157.749 \text { dólares em } \\
\text { comparação com } \\
\text { Fulvestrant e; } 115.624 \\
\text { dólares em comparação } \\
\text { com a tamoxifeno. } \\
\end{array}$ & $\begin{array}{l}\text { Disposição a pagar }=<130.000 / \text { QALY dólares. } \\
\text { O estudo demonstrou que a terapia com Exemestano }+ \\
\text { Everolimus apresenta relação de custo efetividade favorável } \\
\text { quando comparada a monoterapia de Exemestano e } \\
\text { Tamoxifeno. }\end{array}$ \\
\hline A14 & $\begin{array}{l}\text { Hu X and Hay } \\
\text { JW, } 2018\end{array}$ & $\begin{array}{l}\text { Pembrolizumab } \\
\text { (92.833 euros); } \\
\text { Quimioterapia padrão. } \\
\text { (20.368 euros). }\end{array}$ & $\begin{array}{l}\text { Pembrolizumab } \\
(1.54) ; \\
\text { Quimioterapia padrão. } \\
(0.71) .\end{array}$ & $\begin{array}{l}\text { Incremento de } 86.913 \\
\text { euros por QALY para a } \\
\text { Pembrolizumab. }\end{array}$ & $\begin{array}{l}\text { Disposição a pagar }=<50.000 / \text { QALY euros. } \\
\text { O estudo demonstrou que o Pembrolizumab não é rentável no } \\
\text { seu preço de tabela atual e é necessário um desconto de } 50 \% \\
\text { ou mais para que seja rentável em comparação com a } \\
\text { quimioterapia comumente prescrita, sem que com os valores } \\
\text { normas não apresentou relação de custo efetividade favorável. }\end{array}$ \\
\hline A15 & $\begin{array}{l}\text { Zhang } P \text {, et } \\
\text { al., } 2016\end{array}$ & $\begin{array}{l}\text { FOLFOX4 } \\
\text { (6.876 dólares); } \\
\text { Sorafenib } \\
\text { (18.748 dólares). }\end{array}$ & $\begin{array}{l}\text { FOLFOX4 } \\
(0.38) ; \\
\text { Sorafenib } \\
(0.39)\end{array}$ & $\begin{array}{l}\text { Redução de } 934.801 \\
\text { dólares por QALY para o } \\
\text { FOLFOX4. }\end{array}$ & $\begin{array}{l}\text { Disposição a pagar }=<23.970 / \text { QALY dólares. } \\
\text { Na análise probabilística da sensibilidade, a probabilidade de } \\
\text { FOFLOX4 ser uma opção ótima foi de } 100 \% \text { com base na } \\
\text { disposição a pagar chinês, apresentando uma relação de custo } \\
\text { efetividade favorável. }\end{array}$ \\
\hline A16 & $\begin{array}{l}\text { Goldstein DA, } \\
\text { et al., } 2015\end{array}$ & $\begin{array}{l}\text { Gemcitabina + } \\
\text { Cisplatina. ( } 50.205 \text { dólares); } \\
\text { Gemcitabina + Cisplatina + } \\
\text { Necitumumab e manutenção } \\
\text { com Necitumumab ( } 37.173 \\
\text { dólares). }\end{array}$ & $\begin{array}{l}\text { Gemcitabina + } \\
\text { Cisplatina. }(2.03) \\
\text { Gemcitabina + Cisplatina } \\
+ \text { Necitumumab e } \\
\text { manutenção com } \\
\text { Necitumumab (1.46). }\end{array}$ & $\begin{array}{l}\text { Incremento de } 49.493 \\
\text { dólares por QALY para o } \\
\text { Necitumumab se o custo } \\
\text { por ciclo for } 350 \text { dólares. }\end{array}$ & $\begin{array}{l}\text { Disposição a pagar }=<100.000 / \text { QALY dólares. } \\
\text { A redução do custo do Necitumumab para } 350 \text { dólares por } \\
\text { ciclo, faz com que o mesmo apresente uma relação custo } \\
\text { efetividade favorável, porém não apresentou melhoria na } \\
\text { qualidade de vida dos pacientes. }\end{array}$ \\
\hline A17 & $\begin{array}{l}\text { Van Kampen } \\
\text { RJW, et al., } \\
2017\end{array}$ & $\begin{array}{l}\text { Bevacizumabe + Taxanos } \\
\text { (125,496 euros); } \\
\text { Taxanos } \\
(69,282 \text { euros }) .\end{array}$ & $\begin{array}{l}\text { Bevacizumabe }+ \\
\text { Taxanos (1.79); } \\
\text { Taxanos }(1.41)\end{array}$ & $\begin{array}{l}\text { Incremento de } 155.261 \\
\text { euros por QALY para o } \\
\text { Bevacizumabe + } \\
\text { Taxanos }\end{array}$ & $\begin{array}{l}\text { Disposição a pagar }=<80.000 / \text { QALY euros. } \\
\text { De acordo com o limiar informal holandês, o bevacizumab, } \\
\text { além do tratamento com taxano, não foi considerado custo- } \\
\text { efetivo para o câncer de mama metastático. }\end{array}$ \\
\hline A18 & $\begin{array}{l}\text { Carlson J, et } \\
\text { al., } 2017\end{array}$ & $\begin{array}{l}\text { Alectinibe (255,413 dólares); } \\
\text { Ceritinibe (241,545 dólares). }\end{array}$ & $\begin{array}{l}\text { Alectinibe } \\
\text { (1.42); Ceritinibe (0.98). }\end{array}$ & $\begin{array}{l}\text { Incremento de } 31.180 \\
\text { dólares por QALY para o } \\
\text { Alectinibe }\end{array}$ & $\begin{array}{l}\text { Disposição a pagar }=<100.000 / \text { QALY dólares. } \\
\text { O estudo mostra que estimadamente o custo de } 31.180 \\
\text { dólares, faz do Alectinibe uma opção econômica em } \\
\text { comparação ao Ceritinibe nos EUA, com uma relação custo } \\
\text { efetividade favorável. }\end{array}$ \\
\hline
\end{tabular}

Fonte: Silva JAC, 2018.

Legenda: ' ${ }^{Q}$ ALYs: anos de vida ajustados a qualidade; ${ }^{2} I C E R$ : relação custo-benefício incremental. 


\section{Análises de sensibilidade}

Diversos estudos utilizaram a Simulação de Monte Carlo (SMC) para descobrir estratégias de incertezas (Rivera F, et al., 2017; Saito S, et al., 2017; Zhan M, et al., 2017) outros utilizaram curvas de aceitabilidade de custo-efetividade para apresentar os resultados das análises de sensibilidade probabilística (CARLSON JJ, et al., 2017; VAN KAMPEN RJW, et al., 2017; CASADO LF, et al., 2018). Para explorar as possíveis influências de diferentes parâmetros, os autores descreveram os resultados através um diagrama de tornado Saito S, et al. (2017); Zhen H, et al. (2018); Zhou K, et al. (2018) esta metodologia resultou em diversos achados.

Demonstrou-se, em alguns estudos, que a fase do modelo de Markov que mais influenciou nos custos do tratamento foi a fase de doença progressiva (progressive disease - PD), Xie J, et al. (2015); Bohensky MA, et al. (2016); Van Kampen RJW, et al. (2017); Zhou K, et al. (2018), em concordância, no estudo de Zhen H, et al., (2018), foi demonstrado que o aumento do tempo (20\%) na fase livre da doença, representou na redução de quase metade do ICER, em consonância com outros ensaios Zhang P, et al. (2016); Zhan M, et al. (2017); Casado L, et al. (2018); Hu X e Hay JW, (2018), demonstrando ser uma parcela importante nos custos totais (SAITO S, et al., 2017). Hu X e Hay JW, (2018), demonstraram que a inclusão hipotética de cuidados informais aos custos do paciente, é capaz de impactar substancialmente no ICER.

Algumas terapias, devido ao custo elevado, influenciaram diretamente o ICER como é o caso do Bevacizumabe Cohn DE, et al. (2015); Zhan M, et al. (2017); Zhen H, et al. (2018), Sorafenib Zhang P, et al. (2016), Everolimus Xie J, et al. (2015), Nivolumab Bohensky MA, et al. (2016); Sarfaty M, et al. (2018), Ramucirumab (SAITO S, et al., 2017).

Outros critérios, em determinadas pesquisas, superaram os gastos com o tratamento padrão, como é o caso do câncer de pulmão, os custos da internação eram os principais, bem como os custos do tratamento paliativo ou terminal, ocupando quase $65 \%$ do total de gastos (ZHOU K, et al., 2018). Os custos hospitalares representaram grande importância para o ICER, em alguns estudos Zhou K, et al. (2018), em contraponto, o mesmo, não apresentou tal característica (ZHAN M, et al., 2017; ZHEN H, et al., 2018;). Da mesma forma, alguns fatores foram pouco influenciadores no ICER final, como os gastos relacionados aos EAs de grau 3-4 e os gastos indiretos (SAITO S, et al., 2017; ZHAN M, et al., 2017; ZHEN H, et al., 2018).

\section{Qualidade de vida e a Razão de custo-efetividade incremental (ICER)}

O termo "utilidade" refere-se à qualidade percebida pelos pacientes com base em seu estado de saúde e recebe um valor entre 1 (saúde perfeita) e 0 (estado de saúde equivalente à morte) Casado LF, et al. (2018), estes dados, a sobrevivência e o QALY, foram extraídos pelos estudos revisados de ensaios clínicos randomizados, e foram utilizados associados a estimativa dos custos globais, na composição do valor de custo-eficácia incremental (ICER) (ZHEN M, et al., 2018; ZHOU K, et al., 2018). Os dados de efetividade que foram inseridos em cada ciclo de Markov, a partir dos resultados coletados, seguiram a mesma metodologia (MATTER-WALSTRA K, et al., 2015; XIE J, et al., 2015; ZHANG P, et al., 2016).

Os ensaios clínicos randomizados utilizados, em alguns casos, avaliavam diretamente a qualidade de vida (Zhang P, et al., 2016), em outros, utilizavam os valores de pesquisas anteriores, onde esse critério tinha sido avaliado (GOLDSTEIN DA, et al., 2015; RIVERA F, et al., 2017; SAITO S).

Dos 18 (dezoito) trabalhos avaliados nessa revisão, apenas 7 (sete) demonstram como resultado final da análise econômica, um ICER dentro do limite de disposição a pagar, todos, através da comparação entre terapias imunológicas (RIVERA F, et al., 2017; CASADO LF, et al., 2018; SARFATY M, et al., 2018).

De maneira geral, os grupos de terapia combinada de quimioterapia e imunoterapia apresentaram melhor resultado na qualidade de vida, porém não foi demonstrado compatibilidade com o limite de disposição a pagar do estado (VAN KAMPEN RJW, et al., 2017; ZHAN M, et al., 2017; HU X e HAY JW, 2018; ZHEN H, et al., 2018). 
Em diversos estudos a comparação entre as terapias obteve respostas semelhantes na qualidade de vida, entretanto o braço com esquema quimioterápico demonstrou considerável diminuição do ICER (ZHANG P, et al., 2016). Em outras situações, como no caso da inserção de imunoterapia, ocorreu o contrário no ICER (SAITO S, et al., 2017).

\section{DISCUSSÃO}

Este estudo permitiu um panorama global da produção científica sobre as avaliações de custo efetividade no tratamento oncológico, através da relação entre avaliação de custo terapêutico e qualidade de vida dos pacientes. Dentre os 18 (dezoito) artigos que compuseram a amostra, o principal cenário metodológico encontrado, foi a análise econômica, feita pelos autores, de dados oriundos de ensaios clínicos randomizados de fase II/III, mostrando o interesse em maiores pesquisas no âmbito do poder de pagamento público e terceirizado em saúde, associado as novas tecnologias antineoplásicas insurgentes.

A avaliação do curso de uma doença entre os estados de saúde, é construída pelo trânsito de uma coorte hipotética de pacientes e possibilita a avaliação paralela dos gastos diretos e indiretos, uma das formas de construir essa avaliação é através de modelos de Markov, onde o paciente permanece períodos diferentes em cada fase da doença, podendo progredir entre eles, dependendo do estado de saúde (SATO RG e ZOUAIN DM, 2010).

Esta revisão demonstrou que o tempo de permanência em diferentes fases no modelo de Markov, podem influenciar nos gastos, como por exemplo o aumento da permanência no estado de doença progressiva, como apontado por diversos autores Hirschmann A, et al. (2018), em contraponto ao prolongamento do estado livre de progressão da doença (ZHAN M, et al. 2017; CHEN HD, et al., 2017). Este resultado está relacionado a gravidade dos pacientes, ao uso das terapias em doses maiores, comorbidades e reações adversas mais incidentes, na fase em que a doença está em progresso (HIRSCHMANN A, et al., 2018). Sendo assim os autores discutem, que quanto maior for a permanência na fase livre de progressão da doença, menor será o gasto e as intercorrências do tratamento.

É conhecido que a sustentabilidade terapêutica e a qualidade de vida, tornaram-se desfechos primários, em ensaios clínicos que avaliam a eficácia do tratamento para câncer, uma vez que mede o bem-estar geral dos pacientes, particularmente em pacientes com doenças avançadas (BOTTOMLEY A, et al., 2002). Outro elemento na sustentabilidade (financeira) do tratamento do câncer nos sistemas de saúde, é o custo do número crescente de tratamentos e drogas inovadoras (LUENGO-FERNANDEZ R, et al., 2013). Além disso, nas avaliações recentes do custo terapêutico real, são apontadas diferenças substanciais de preço nos medicamentos em diferentes países europeus Vogler S, et al. (2016), assim como demonstrou essa revisão, através da disparidade dos custos dos fármacos dependendo do estudo e do WTP utilizado.

Os custos diretos de saúde, como os associados ao diagnóstico, estadiamento da doença e manejo dos pacientes com câncer, são fatores importante que compõem a fonte de morbidade e mortalidade da doença Goodwin RJ e Sheperd FA, (2000), os mesmos estiveram presentes nos estudos revisados, onde foram apontados gastos como custo das drogas, da administração das mesmas, exames, tratamentos para Eventos adversos (EAs) e hospitalização (CARLSON JJ, et al., 2017, VAN KAMPEN RJW, et al., 2017).

Já os custos indiretos, apresentam uma dificuldade natural na aproximação do gasto ao indivíduo, da apropriação correta do custo por paciente, exigindo critérios de atribuição para serem melhor entendidos. Sendo assim, gastos de transporte, estadia, previdência e afastamento, dentre outros, ganham importância apenas na perspectiva do pagamento, como demonstrado nesta revisão, onde todos os artigos que declararam utilizar a perspectiva de pagamento de terceiros utilizaram apenas gastos diretos, Matter-Walstra K, et al. (2015); Miguel LS, et al. (2017); Rivera F, et al. (2017); Hu X e Hay JW, (2018), sendo que os únicos artigos que fizeram menção a gastos indiretos, avaliaram taxas de viagem Zhou K, et al. (2018) e os dias em que os pacientes ficaram sem poder trabalhar (ZHANG P, et al., 2016). 
Nas avaliações econômicas, encontrou-se predominância de ensaios comparativos entre braços com tratamentos imunoterápico e com quimioterapia padrão Cohn DF, et al., (2015); Goldstein DA, et al., (2015); Zhang P, et al., (2016), na tentativa de justificar através dos ganhos no QALYS, a inserção de terapias inovadoras, do ponto de vista farmacológico, mas onerosas do ponto de vista do ICER.

Neste ponto, algumas das avaliações econômicas, obtiveram sucesso Rivera F, et al. (2017); Goldstein DA, et al. (2015), ao demonstrar que, apesar de associar à terapia padrão, ou até substituir a mesma, por uma nova opção terapêutica, não necessariamente faz-se presente o aumento do ICER além do limite do WTP, demonstrando uma relação custo-efetividade favorável.

Entretanto, em sua maioria, a revisão dos estudos demonstrou que ocorre um incremento muito alto por ano de QALY, com a inserção da imunoterapia Cohn DE, et al., (2015); Saito S, et al. (2017); Van Kampen RJW, et al. (2017), principalmente, devido ao alto preço dos medicamentos Cohn DE, et al., (2015); Saito S, et al. (2017); Zhan M, et al. (2017); Zhen H, et al. (2018), como já publicado na literatura sobre o tema (ZHOU K, et al., 2018).

Diversos estudos demonstraram que os agentes quimioterápicos são os que mais influenciam nos custos do tratamento, sendo que a introdução de apresentações genéricas e/ou frascos de tamanho intermediário, são capazes de reduzir o custo incremental (ZHOU K, et al., 2018). Nenhum artigo pesquisado avaliou esse tipo de mudança, mas sim, alterações no esquema terapêutico, como Hu X e Hay JW, (2018), demonstram que se houvesse um desconto de $50 \%$ no preço da terapia imunológica, a mesma, passaria a deter de uma relação custo-efetividade favorável ao seu uso.

Goldstein DA, et al. (2015), descreveram que em caso de diminuição o custo do imunoterápico em cinco vezes, seria possível uma relação custo-efetividade favorável, assim como em diversos outros estudos (COHN DE, et al., 2015).

As imunoterapias emergiram rapidamente como ferramentas importantes no arsenal oncológico. Embora englobando uma diversidade de agentes que atuam no sistema imunológico antitumoral (por exemplo, anticorpos monoclonais, moléculas pequenas, vacinas e terapias virais ou celulares), as imunoterapias mais utilizadas são o checkpoint imunológico inibidores (ICls) (VERMA V, et al., 2018). Esses agentes tornaramse o padrão de cuidados em circunstâncias clínicas apropriadas, com base de numerosos ensaios clínicos, porém existem controvérsias quanto a viabilidade econômica dessas terapias (PRASAD V e GRADY C, 2014).

A comparação entre terapias imunológicas se fez presente nesta revisão Rivera F, et al. (2017); Casado LF, et al. (2018); Sarfaty M, et al. (2018) e, neste caso, apresentando benefícios nos critérios de custoefetividade, quando comparadas as novas terapias com as terapias imunológicas já existente, como já presente na literatura (VALENCIA JJV e ESQUEDA MEA, 2015).

Várias hipóteses podem ser construídas, sendo que uma terapia padrão onerosa faz com que o novo medicamento pareça rentável devido a apresentar um ICER relativamente baixo Bach PB (2015), a exemplo dos casos relatados em nossa revisão, como Sarfaty M, et al., (2018) que demonstraram custo-efetividade na em comparação a terapia padrão, porém não ao placebo. Este fato é devido ao alto ICER que a terapia padrão detém, mascarando o ICER do Nivolumab. O mesmo pode ter acontecido no estudo de Rivera F, et al., 2017, onde o Panitumumabe apresentou-se com melhor relação custo-efetividade que o Bevacizumabe, outro fármaco do alto custo, no estudo de Miguel L et al., 2017, com o fármaco Pembrolizumab e no estudo de Carlson JJ et al. (2017), com o fármaco Alectinibe.

Além disso, a terapia padrão muda rapidamente, portanto, estudos diferentes para a mesma indicação devem ser feitos, antes de qualquer tomada de decisão (PRASAD V e GRADY C, 2014). Muitos vezes os medicamentos inovadores são aprovados com base em dados de sobrevida imaturos, mas significativamente superiores, para facilitar o acesso precoce dos pacientes, extrapolando a sobrevida dos pacientes, baseado em um tempo de acompanhamento mais curto e, portanto, os resultados podem ser influenciados pelos pressupostos de um modelo com tempo maior (HU X e HAY JW , 2018). 
Métodos diferentes são usados na tomada de decisões de cobertura na saúde pública. O Reino Unido usa a rentabilidade, assim como o Canadá e alguns outros países da Europa (GOLDSTEIN DA e SARFATY M, 2016). Notavelmente, nos EUA, a FDA não leva em consideração o custo, a Medicare, por lei, fornece qualquer medicamento aprovado pela FDA, independentemente do preço.

Boa parte dos autores demonstraram que mesmo a inserção de uma nova opção terapêutica apresentando uma melhora na qualidade de vida dos pacientes, representava um ICER superior ao limite de custoefetividade da disposição a pagar do país de estudo, não apresentando uma relação custo efetividade favorável (COHN DE, et al., 2015; ZHOU K, et al., 2018; MATTER-WALSTRA K, et al., 2015; ZHEN H, et al., 2018).

Embora, em alguns casos, os mesmos valores de ICER sendo comparados a WTP de países diferentes, onde a disposição de pagar é maior, pode tornar custo-efetiva uma opção terapêutica, que em um primeiro momento, era desfavorável economicamente (HU X e HAY JW, 2018) como no estudo de Sarfaty M, et al., 2018, que demonstrou que o emprego do Nivolumab na terapia antineoplásica, seria $90 \%$ custo-efetivo se tiver aumento na disposição a pagar para 140.000/QALY dólares. Demonstrando que o preço das terapias e a disposição a pagar de cada país, ainda são fatores limitantes na sustentabilidade das terapias.

\section{CONCLUSÃO}

Em geral, os estudos que integraram a presente revisão abordaram a problemática comum das publicações sobre custo efetividade nos tratamentos para o câncer. Apesar das limitações relacionadas aos vieses de seleção, desempenho e detecção, a amostragem representou um panorama das publicações sobre o tema, emergindo conclusões importantes.

A dificuldade em comprovar através de análise de custo efetividade o emprego de novas terapias, foi notável, dentre as publicações. O que gerou, o esforço dos autores em tentar, através de opções diferenciadas no esquema terapêutico e outros fatores, reduzir o custo das drogas e assim alcançar a sustentabilidade compara com o limite de disposição a pagar de cada país. O método de composição dos modelos de Markov, como base para as avaliações econômicas, apresentou certa linearidade, conceituando-se como um mecanismo interessante, para estudos em saúde. Sendo assim, com esses dados, pode-se afirmar que são importantes novas revisões, focadas separadamente em cada tipo de neoplasia, a fim de construir uma base confiável de informações para tomada de decisão terapêutico e econômica, tanto para o âmbito público como privado.

\section{REFERÊNCIAS}

1. BACH PB. New math on drug cost-effectiveness. N Engl J Med, 2015; 373:1797-9.

2. BLOOM DE, et al. The Global Economic Burden of Non-communicable Diseases. Geneva: World Economic Forum, 2011.

3. BOHENSKY MA, et al. A Cost-Effectiveness Analysis of Nivolumab Compared with Ipilimumab for the Treatment of BRAF Wild-Type Advanced Melanoma in Australia. Value Heal, 2016; 19(8): 1009-15.

4. BOTTOMLEY A. The cancer patient and quality of life. Oncologist, 2002; 7:120-25.

5. BRASIL. Ministério da Saúde. Debater sobre oncologia. 2015b. Disponível em: http://www2.camara.leg.br/atividadelegislativa/comissoes/comissoes-permanentes/csst/audiencias-publicas/audiencia-publica-2015/aud-publica-2508/apresentacao-paulo-eduardo-xavier-mendonca. Acesso em: 03 nov. 2018.

6. CARLSON JJ, et al. The cost-effectiveness of alectinib in anaplastic lymphoma kinase-positive (ALK+) advanced NSCLC previously treated with crizotinib. J Med Econ, 2017; 20(7): 671-7.

7. CASADO LF, et al. Papr reduction using scs-slm technique in stfbc mimo-ofdm. Phrmacoeconomics Outcomes Res Iberia. 2017; 12(10): 3218-21.

8. CHEN HD, et al. Cost-effectiveness analysis of apatinib treatment for chemotherapy-refractory advanced gastric cancer. J Cancer Res Clin Oncol, 2017; 143(2): 361-68.

9. COHN DE, et al. A cost-utility analysis of NRG Oncology/Gynecologic Oncology Group Protocol 218: Incorporating prospectively collected quality-of-life scores in an economic model of treatment of ovarian cancer. Gynecol Oncol, 2015; 136(2): 293-9.

10. GOLDSTEIN DA, et al. Necitumumab in metastatic squamous cell lung cancer. JAMA Oncol, 2015; 1(9): $1293-300$. 
11. GOLDSTEIN DA, SARFATY M. Cancer drug pricing and reimbursement: lessons for the United States from around the world. Oncologist, 2016; 21:907-9.

12. GOODWIN PJ, SHEPHERD FA. Economic issues in lung cancer: a review. J Clin Oncol, 2000; 16(12): 3900-12.

13. HALL M, LLOYD H. Evaluating patients' experiences of home and hospital chemotherapy. Cancer Nurs. Pract. 2008; 7(1): 35-8.

14. HETTLE R, et al. Challenges in economic modeling of anticancer therapies: an example of modeling the survival benefit of olaparib maintenance therapy for patients with BRCA-mutated platinum-sensitive relapsed ovarian cancer. J Med Econ, 2015; 18:516-24

15. HIGGINS JP, et al. The Cochrane Collaboration's tool for assessing risk of bias in randomised trials. BMJ, $2011 ; 343: \mathrm{d} 5928$.

16. HIRSCHMANN A, et al. Swiss Group of Clinical Cancer Research (SAKK). Cost-effectiveness of nivolumab in the treatment of head and neck cancer. Oral Oncol. 2018; 87:104-10.

17. HU X, HAY JW. First-line pembrolizumab in PD-L1 positive non-small-cell lung cancer: A cost-effectiveness analysis from the UK health care perspective. Lung Cancer, 2018; 123:166-71.

18. INCA. Ministérios da Saúde. Coordenação Geral de Ações Estratégicas. Coordenação de Prevenção e Vigilância. Estimativa 2016: Incidência de câncer no Brasil, Rio de Janeiro. Disponível em: http://www.inca.gov.br/estimativa/2014/. Acesso em: 02 nov. 2018.

19. LEAL L, Et al. Análise de custo-efetividade da abiraterona para câncer de próstata metastático resistente à castração em pacientes com uso prévio de quimioterapia. J Bras Econ da Saúde, 2018; 10(2): 126-33.

20. LUENGO-FERNANDEZ R, et al. Economic burden of cancer across the European Union: a population-based cost analysis. Lancet Oncol, 2013; 14:1165-74.

21. MATTER-WALSTRA $\mathrm{K}$, et al. A cost-effectiveness analysis of trametinib plus dabrafenib as first-line therapy for metastatic BRAF V600-mutated melanoma in the Swiss setting. Br J Dermatol, 2015; 173(6): 1462-70.

22. MIGUEL LS, et al. Cost Effectiveness of Pembrolizumab for Advanced Melanoma Treatment in Portugal. Value Heal, 2017; 20(8): 1065-73.

23. MOHER D, et al. Preferred reporting items for systematic reviews and meta-analyses: the PRISMA statement. PLoS Med, 2010: 8;336-41.

24. PRASAD V, GRADY C. The misguided ethics of crossover trials. Con- temp Clin Trials, 2014; 37(2): 167-69.

25. RIVERA F, et al. Cost-effectiveness analysis in the Spanish setting of the PEAK trial of panitumumab plus mFOLFOX6 compared with bevacizumab plus mFOLFOX6 for first-line treatment of patients with wild-type RAS metastatic colorectal cancer. J Med Econ, 2017; 20(6): 574-84.

26. SAITO S, et al. Cost-effectiveness of Paclitaxel + Ramucirumab Combination Therapy for Advanced Gastric Cancer Progressing After First-line Chemotherapy in Japan. Clin Ther, 2017; 39(12): 2380-8.

27. SARFATY M, et al. Cost Effectiveness of Nivolumab in Advanced Renal Cell Carcinoma. Eur Urol, 2018; 73(4): 628-34.

28. SATO RC, ZOUAIN DM. Modelos de Markov aplicados a saúde. Einstein, 2010; 8(3): 376-9.

29. SECOLI SR, et al. Avaliação de tecnologia em Saúde II. A análise de custo-efetividade. Arq Gastroenterol, 2010; 47(4): 329-33.

30. SOAREZ PC, NOVAES HMD. Limiares de custo-efetividade e o Sistema Único de Saúde. Cad. Saúde Pública, 2017; 30(4): e00040717.

31. VALENCIA JJV, ESQUEDA MEA. Análisis de costo-efectividad de panitumumab + FOLFOX en CCRm RAS-WT, Gaceta Mexicana de Oncología, 2015; 14(5): 250-58.

32. VAN KAMPEN RJW, et al. Real-world and trial-based cost-effectiveness analysis of bevacizumab in HER2-negative metastatic breast cancer patients: a study of the Southeast Netherlands Breast Cancer Consortium. Eur J Cancer, 2017; 79:238-46.

33. VERMA V, et al. A systematic review of the cost and cost effectiveness studies of immune checkpoint Inhibitors. Journal for Immuno Therapy of Cancer, 2018; 6:128.

34. VOGLER S, et al. Cancer drugs in 16 European countries, Australia, and New Zealand: a cross-country price comparison study. Lancet Oncol, 2016;17:39-47.

35. WHO. Global Health Estimates 2015: deaths by cause, age, sex, by country and by region, 2000-2015. 2016; Disponível em: http://www.who.int/healthinfo/global_burden_disease/estimates/en/index1.html. Acesso em: 02 nov. 2018.

36. XIE J, et al. Economic Evaluations of Everolimus Versus Other Hormonal Therapies in the Treatment of HR+/HER2Advanced Breast Cancer from a US Payer Perspective. Clin Breast Cancer, 2015; 15(5): 263-76.

37. ZHAN M, et al. Cost-effectiveness analysis of additional bevacizumab to pemetrexed plus cisplatin for malignant pleural mesothelioma based on the MAPS trial. Lung Cancer, 2017; 110:1-6.

38. ZHANG $P$, et al. FOLFOX4 or sorafenib as the first-line treatments for advanced hepatocellular carcinoma: A costeffectiveness analysis. Dig Liver Dis, 2016; 48(12): 1492-7.

39. ZHEN H, et al. Cost-effectiveness analysis of the addition of bevacizumab to chemotherapy as induction and maintenance therapy for metastatic non-squamous non-small-cell lung cancer. Clin Transl Oncol, 2018; 20(3): 286-93.

40. ZHOU K, et al. Cost-effectiveness analysis of sensitive relapsed small-cell lung cancer based on JCOG0605 trial. Clin Transl Oncol, 2018; 20(6): 768-74. 